\title{
Duodenal and pancreas metastasis from lung cancer
}

\author{
Tomohiro TAMURA ${ }^{1}$ \\ Gen OHARA ${ }^{1}$ \\ Katsunori KAGOHASHI ${ }^{1}$ \\ Norio TAKAYASHIKI ${ }^{1}$ \\ Hiroaki SATOH ${ }^{1}$
}

${ }^{1}$ Department of Respiratory, Mito Medical Center, Tsukuba University,
Mito, Japan
${ }^{1}$ Tsukuba Üniversitesi Mito Tıp Merkezi, Solunum Bölümü, Mito, Japonya
Distant metastases from lung cancer are usually found in the lung, bone, brain, liver, and adrenal gland; however, metastases in duodenum and pancreas are very rare (1-10). We report herein our experience with case of duodenal and pancreas metastases from lung adenocarcinoma.

A 56-year-old man was referred to our hospital due to an ill-defined mass, which was diagnosed as having lung adenocarcinoma pathologically, in the left lower lobe of the lung with ipsilateral mediastinal lymph nodes (Figure 1). The patient was treated with platinum-based chemotherapy for 5 months. The patient was readmitted with the two weeks history of epigastric pain. He also complained of anorexia, lethargy, and weight loss of 4 kilograms over past four months. Epigastric pain was not dull in nature, aggravated by food in take, and it had increased in intensity over two weeks, for which he was taking oxycodone/acetaminophen, but minimal pain relief. The patient had 10-year history of systemic lupus erythematosus, which has been treated with prednisolone. He was ex-smoker with one pack a day for 20 years. On physical examination, he was found in fair general condition, but his vitals were stable.
On abdominal examination, there was mild epigastric tenderness without any rigidity, guarding, orrebound tenderness. The rest of systemic examination was unremarkable. Complete blood count (CBC) showed hemoglobin $12.0 \mathrm{gm} / \mathrm{dL}$, white blood cells (WBC) 8200/microL; and platelets 336.000/microL. Liver and renal function tests were within normal limits. Fecal occult blood (FOB) test was found negative. Upper gastrointestinal endoscopy revealed a tumorous lesion at the opposite site of papilla of Vater (Figure 2). Pathological specimen from the duodenal lesion showed adenocarcinoma and immuno histochemical staining for thyroid transcription factor-1 positive cells (Figure 3A: Hematoxylin-Eosin staining, Figure 3B: thyroid transcription factor-1: TTF-1). These findings strongly supported the diagnosis of metastatic adenocarcinoma of the lung origin. Computed tomography (CT) of abdomen showed ill-defined mass measuring $3.2 \times 3.0 \mathrm{~cm}$ in

\section{Yazışma Adresi (Address for Correspondence)}

Dr. Hiroaki SATOH

University of Tsukuba, Mito Medical Center, Department of Respiratory Medicine, TSUKUBA - JAPAN

e-mail: hirosato@md.tsukuba.ac.jp 


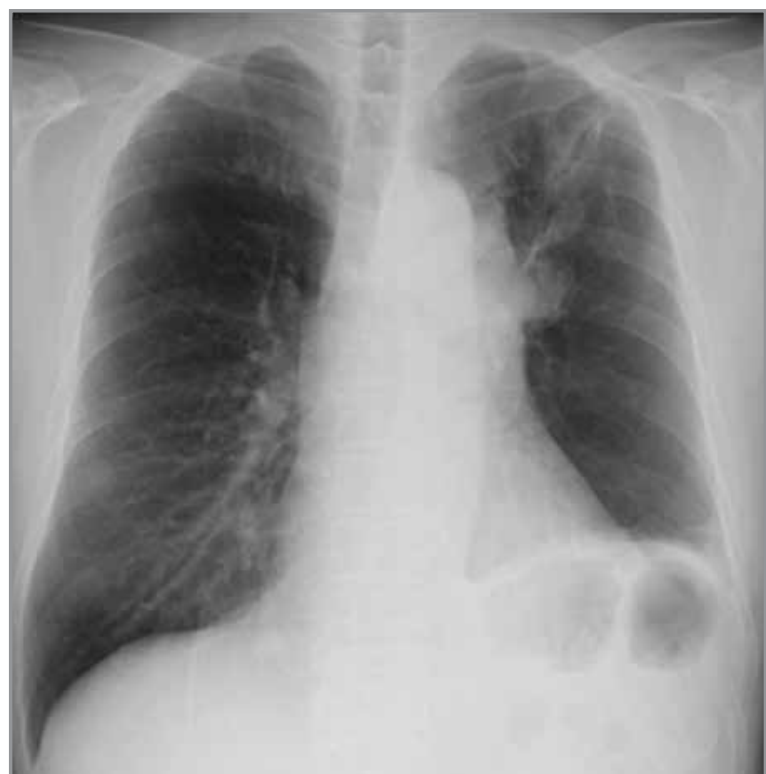

Figure 1. Chest radiograph on admission shows an ill-defined mass, which was diagnosed as having lung adenocarcinoma pathologically, in the left lower lobe of the lung with ipsilateral mediastinal lymph nodes.

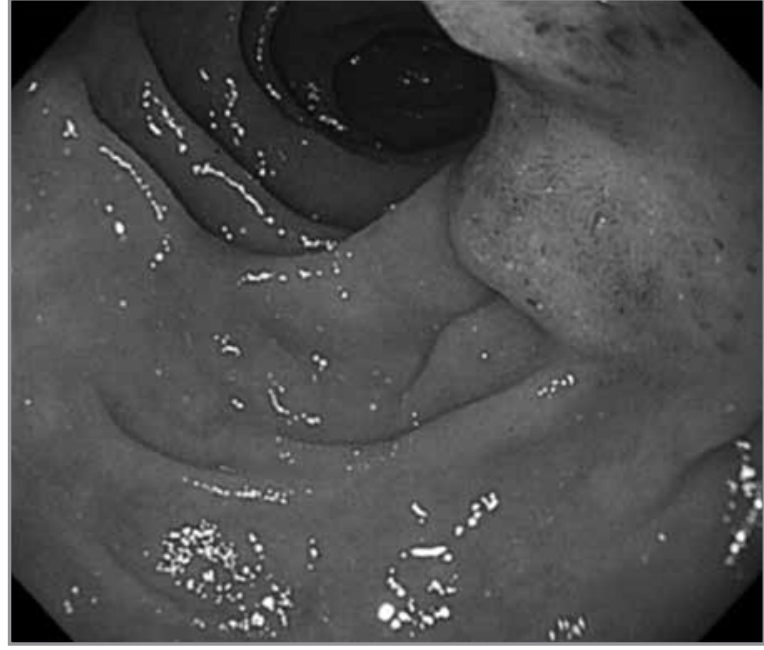

Figure 2. Upper gastrointestinal endoscopy revealed a tumorous lesion at the opposite site of papilla of Vater.
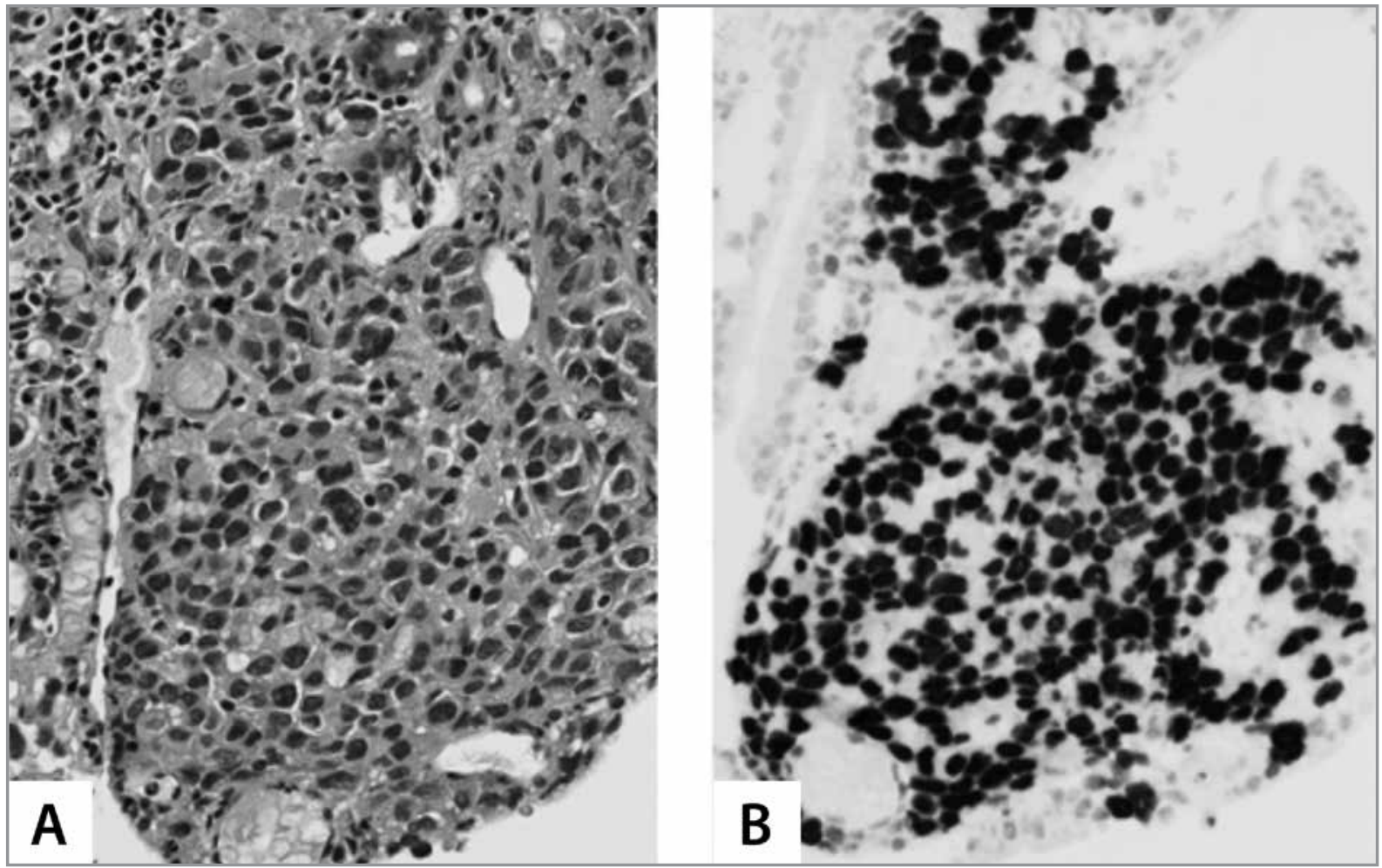

Figure 3. Pathological specimen from the duodenal lesion showed adenocarcinoma and immunohistochemical staining for thyroid transcription factor-1 positive cells. (A) Hematoxylin-Eosin staining, (B) thyroid transcription factor-1. 
pancreas body, and two intrahepatic metastatic lesions, which were not detected in abdominal CT scan at the time of initial diagnosis of lung adenocarcinoma. CT-positron emission tomography (CT-PET) showed 18flouro-deoxyglucose (FDG) avid a mass in pancreas body [standardize duptake volume (SUVmax)7.45] and a mass in the left lower lobe of the lung [(SUVmax) 20.8] (Figure 4). ERCP was not performed considering metastatic condition in other sites and refusal of the patient. The patient had rapidly progressive disease and died of 2 months after the diagnosis of these metastases.

Patients with abdominal organ metastases from lung cancer have several symptoms (1-10). Among them, patients with gastrointestinal and pancreatohepatobiliary tract metastases exhibit symptoms such as nausea, vomiting, abdominal pain, appetite loss and weight loss, although some of them are asymptomatic (2). These symptoms can also be observed in lung cancer patients with chemotherapy. In our patient, we first evaluated that nausea, vomiting, epigastric pain, and appetite loss were chemotherapy-induced adverse events because intraabdominal metastasis were not found in the initial evaluation and rarity of the metastases to duodenum and pancreas.

Among all duodenal metastasis, the second portion (descending portion) is most frequent $(58.7 \%)$ as observed in our case (11). As for morphological features of duodenal metastasis, Miyakawa et al. proposed four types of them: compression, protrusion, ulcerand stenosis (11). Appearance of a metastatic lesion fromextra-abdominal primary cancer is all protrusion type with central ulceration, so called 'bull's-eye' (11). This type of metastatic lesion is probably hematogenous. The gross appearance of our case was protrusion type, but having no central ulcer and resembling a submucosal tumor. It is important to differentiate between primary and metastatic duodenal adenocarcinoma to make an appropriate choice of therapy, although it is difficult on occasion $(1,12,13)$. Recently, TTF- 1 has become one of the best immunohistochemical markers to determine the lung orthyroid gland origin and lung (12-15). However, it must be regarded that TTF-1 is expressed frequently only in small cell carcinoma (85\%-90\%) and in adenocarcinoma (75\%-80\%). 14 Sensitivity of TTF-1 for primary lung adenocarcinoma is 57.5 to $76 \%$ and the specificity is almost $100 \% .13$ Thus, TTF- 1 is aspecific marker for these two histopathological types of lung cancer to determine the lung origin in duodenal metastasis, as shown in our case.

With regard to the incidence of pancreas metastasis from lung cancer, we previously studied it in consecutive lung cancer patients in our institution (16). In the report, we showed $26(3.1 \%)$ of 850 patients with primary lung cancer developed synchronous or metachronous pancreatic metastasis; $10.5 \%$ of patients with small cell carcinomas, only $2.3 \%$ of patients with adenocarcinomas, and we concluded that pancreatic metastasis from pulmonary adenocarcinoma was comparatively rare in comparison with small cell carcinoma of the lung (16). Differential diagnosis between a metastatic and a primary pancreatic tumor on the basis of only imaging modalities including $\mathrm{CT}$, endoscopic retrograde cholangiopancreatography or ultrasonography is not easy, especially when a solitary mass isobserved $(8,9)$. Most cases of pancreatic metastasis from lung cancer are unresectable at the
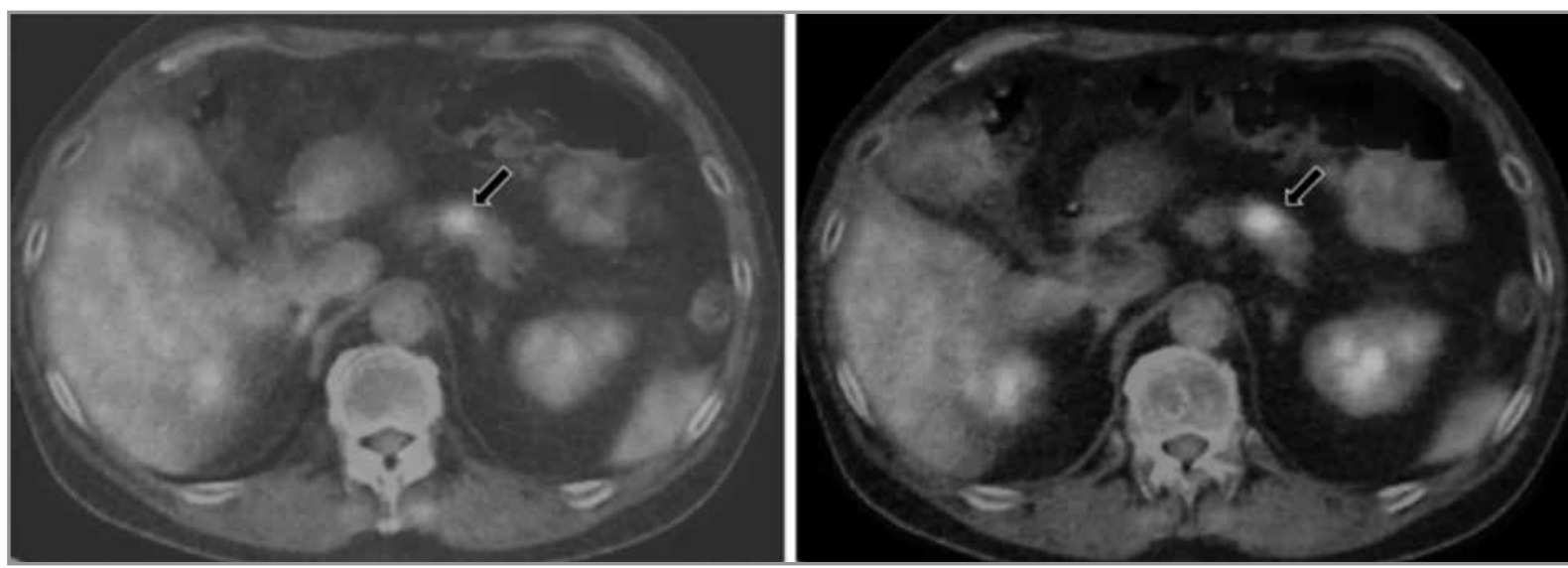

Figure 4. Flouro-deoxyglucose/CT scanshowed ill-defined mass measuring $3.2 \times 3.0 \mathrm{~cm}$ in pancreas body (arrows). 
time of diagnosis because the disease is already widespread. Isolated pancreatic metastases from pulmonary adenocarcinoma without widespread disease are extremely rare. It might be the possibility that the pancreatic lesion was a primary pancreatic tumor. As there was no abnormal finding in abdominal CT scan at the time of initial diagnosis of lung adenocarcinoma, we evaluated it as a solitary pancreas metastasis. In addition, it is interesting that our present case had only intra-abdominal metastases, but had no other metastatic site than abdomen.

Duodenal and pancreatic metastasis from lung adenocarcinoma is extremely rare. To our best knowledge, there has been no such a metastasis as initial presentation or in the clinical course. Taking our experience into consideration, physicians should recognize the possibility of the development of duodenal and pancreas metastasis not only at the time of diagnosis and intheir clinical courses, although it is very rare. If these lesions, whether it may be typical or not, are found in lung cancer patients, obtaining pathological specimens from the lesion and immunohistochemical examination using TTF-1 would be consideredto perform.

\section{REFERENCES}

1. Steinhart AH, Cohen LB, Hegele R, Saibil FG. Upper gastrointestinal bleeding due to superior mesenteric artery to duodenum fistula: rare complication of metastatic lung carcinoma. Am / Gastroenterol 1991;86:771-4.

2. Mosier DM, Bloch RS, Cunningham PL, Dorman SA. Small bowel metastases from primary lung carcinoma: a rarity waiting to be found? Am Surg 1992;58:677-82.

3. Hinoshita E, Nakahashi H, Wakasugi K, Kaneko S, Hamatake $M$, Sugimachi K. Duodenal metastasis from large cell carcinoma of the lung: report of a case. Surg Today 1999;29:799-802.

4. Cremon C, Barbara G, De Giorgio R, Salvioli B, Epifanio G, Gizzi G, et al. Upper gastrointestinal bleeding due to duodenal metastasis from primary lung carcinoma. Dig Liver Dis 2002;34:141-3.
5. Goh BK, Teo MC, Chng SP, Tan HW, Koong HN. Upper gastrointestinal bleed secondary to duodenal metastasis: a rare complication of primary lung cancer. I Gastroenterol Hepatol 2006;21:486-7.

6. Miura T, Shimaoka Y, Nakamura J, Yamada S, Miura T, Yanagi $M$, et al. TTF-1 is useful for primary site determination in duodenal metastasis. World J Gastrointest Oncol 2010;2:360-3.

7. AlSaeed EF, Tunio MA, AlSayari K, AlDandan S, Riaz K. Duodenal metastasis from lung adenocarcinoma: A rare cause of melena. Int J Surg Case Rep 2015;13:91-4

8. Swensen T, Osnes M, Serck-Hanssen A. Endoscopic retrograde cholangio-pancreatography in primary and secondary tumours of the pancreas. Br I Radiol 1980;53:760-4.

9. Wernecke K, Peters PE, Galanski M. Pancreatic metastases: US evaluation. Radiology 1986;160:399-402.

10. Mori N, Sawada T, Satoh H, Kawaguchi M, Hara $H$, Matsushita K. A resected case of solitary pancreatic metastasis from adenocarcinoma of the lung. JOP 2008;9:698-703.

11. Miyakawa K, Sato N, Tateishi U, Inuma H, Moriyama N. Morphological features of metastasis: Duodenum (in Japanesewith English abstract). Stomach and Intestine 2003;38:1790-8.

12. Di Loreto C, Di Lauro V, Puglisi F, Damante G, Fabbro D, Beltrami CA. Immunocytochemical expression of tissue specifictranscription factor-1 in lung carcinoma. J Clin Pathol 1997;50:30-2.

13. Reis-Filho JS, Carrilho C, Valenti C, Leitao D, Ribeiro CA, Ribeiro SG, et al. Is TTF1 a good immuno histochemical marker to distinguish primary from metastatic lung adenocarcinomas? Pathol Res Pract 2000;196:835-40.

14. Moldvay J, Jackel M, Bogos K, Soltész I, Agócs L, Kovács G, et al. The role of TTF-1 in differentiating primary andmetastatic lung adenocarcinomas. Pathol Oncol Res 2004; 10:85-8.

15. Lau SK, Luthringer DJ, Eisen RN. Thyroid transcription factor-1: a review. Appl Immunohistochem Mol Morphol 2002; 10:97-102.

16. Maeno $T$, Satoh $H$, Ishikawa $H$, Yamashita $Y T$, Naito $T$, Fujiwara $M$, et al. Patterns of pancreatic metastasis from lung cancer. Anticancer Res 1998;18:2881-4. 